\title{
Essential fatty acids: theoretical aspects and treatment implications for schizophrenia and depression
}

\author{
Malcolm Peet
}

Almost all current research into developing new pharmacological treatment for depression and schizophrenia is focused directly on modulating neurotransmitter receptors in the brain. This approach was stimulated by the chance discovery of the prototype psychotropic drugs such as chlorpromazine and imipramine and the subsequent development of hypotheses based on the presumed mode of action of these drugs. Even clozapine was first introduced as yet another dopamine receptor blocker, and it was only after it was recognised that clozapine shows improved efficacy that hypotheses were developed as to its possible mode of action. Subsequent research based on the receptor approach has led to improved side-effect profiles for modern psychotropic agents, but has not resulted in any improvement of efficacy over and above that of the drugs discovered by good fortune rather than by hypotheses.

There are only two ways to develop truly novel treatments: to await another stroke of luck or to develop new hypotheses that predict potential treatments. Hypotheses relating to abnormalities of fatty acid and phospholipid metabolism in depression and schizophrenia have given rise to potential new treatment approaches.

\section{What are essential fatty acids?}

All cell membranes, including those of neurons, are composed of a double layer of phospholipid, in which are embedded various functional proteins, including neurotransmitter receptors. Each phospholipid molecule is made up from a 3-carbon glycerol backbone (see Fig. 1). At the Sn3 position are a phosphorus atom and a head group (choline, ethanolamine, inositol or serine). The properties of the phospholipids depend on the nature of the head group and the fatty acids attached at the $\mathrm{Sn} 1$ and $\mathrm{Sn} 2$ positions. There are a wide range of saturated and unsaturated fatty acids and the physical characteristics of the phospholipid molecule, and hence the membrane, vary considerably according to the actual fatty acids attached to the molecule. Altering the lipid environment in the phospholipid bilayer has functional consequences on the activity of the receptors and other proteins embedded in the membrane phospholipid (see Fenton et al, 2000).

Fatty acids can be broadly divided into saturated, monounsaturated and polyunsaturated. The polyunsaturated fatty acids (PUFAs) have the most functional significance and are divided into two main types: the $\mathrm{n} 6$ and the $\mathrm{n} 3$, as shown in Fig. 2 . The distinction between $\mathrm{n} 3$ and $\mathrm{n} 6$ relates to the

$$
\begin{array}{ll}
\text { Sn1 } & \mathrm{CH}_{2} \mathrm{O}-\text { fatty acid } \\
\text { Sn2 } & \stackrel{\mathrm{CHO}}{\mathrm{I}} \text { - fatty acid } \\
\mathrm{Sn} 3 & \mathrm{CH}_{2} \mathrm{O}-\mathrm{P}-\text { head group }
\end{array}
$$

Fig. 1 The general structure of a phospholipid. Sn1, Sn2 and Sn3 denote carbon atoms.

Malcolm Peet is currently Consultant in charge of the Rehabilitation and Assertive Outreach Services for Rotherham Priority Health NHS Trust (Swallownest Court Hospital, Aughton Road, Sheffield S26 4TH, UK; tel: 0114 2872570; e-mail: malcolmpeet@Yahoo.com). He is also Professor Associate at the School of Health and Related Research, University of Sheffield. Clinical interests include the management of treatment-resistant schizophrenia and service development. He has an extensive background of research into the biology and treatment of mental health problems, and his current research interest is in the role of omega-3 polyunsaturated fatty acids in the aetiology and treatment of schizophrenia and depression. 


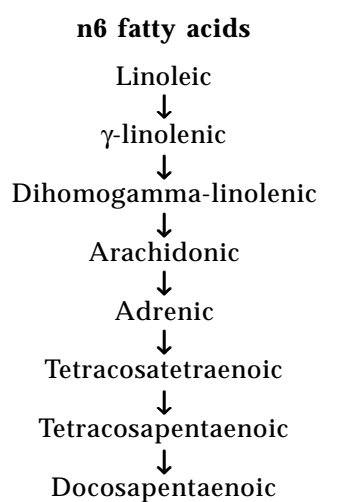

$18: 2$
$18: 3$
$20: 3$
$20: 4$
$22: 4$
$24: 4$
$24: 5$
$22: 5$

$\begin{array}{cc}\text { n3 fatty acids } & \\ \alpha \text {-linolenic } & 18: 3 \\ \downarrow & \\ \text { Octadecatetraenoic } & 18: 4 \\ \downarrow & \\ \text { Eicosatetraenoic } & 20: 4 \\ \downarrow & \\ \text { Eicosapentaenoic } & 20: 5 \\ \downarrow & \\ \text { Docosapentaenoic } & 22: 5 \\ \downarrow & \\ \text { Tetracosapentaenoic } & 24: 5 \\ \downarrow & \\ \text { Tetrahexaenoic } & 24: 6 \\ \downarrow & \\ \text { Docosahexaenoic } & 22: 6\end{array}$

Fig. 2 Summary of the synthesis of essential fatty acids from the dietary precursors linoleic acid (n6 series) and $\alpha$-linolenic acid ( $\mathrm{n} 3$ series). The numbers preceding and following the colons refer to the number of carbons (18-24) and of double bonds (2-6), respectively.

position of the first double bond in the carbon chain. The more double bonds, the more unsaturated the fatty acid. Arachidonic acid and docosahexaenoic acid (DHA) are the most abundant fatty acids in the brain. Arachidonic acid, dihomogamma-linolenic acid and eicosapentaenoic acid (EPA) are also important as cell-signalling and enzyme-regulating molecules and as precursors of eicosanoids (prostaglandins, thromboxanes and leukotrienes). The chemical structure and nomenclature of PUFAs are illustrated in Fig. 3. The parent fatty acids, linoleic (n6) and $\alpha$-linolenic (n3), have been called 'essential' fatty acids because they cannot be synthesised in humans. Although established metabolic pathways exist for production of the other n3 fatty acids, many of them are primarily obtained from marine sources, although the precursor $\alpha$ linolenic acid is present in some vegetable sources,

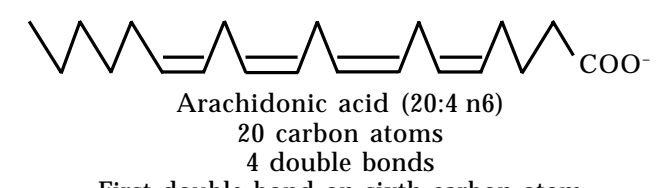

First double bond on sixth carbon atom

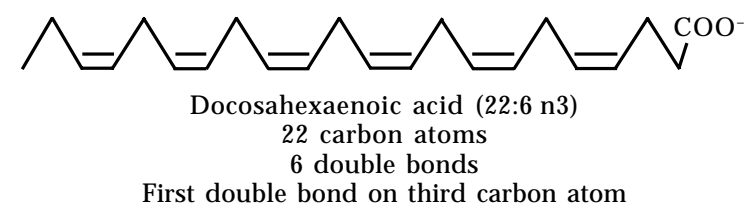

Fig. 3 Chemical struture of arachidonic acid and decosahexaenoic acid, the two most abundant PUFAs in the brain. particularly linseed (flax) oil. The n6 fatty acids come mainly from animal and plant sources.

PUFAs make up 15-30\% of the dry weight of the brain, with arachidonic acid and DHA constituting $80-90 \%$ of that total, of which arachidonic acid is about two-thirds and DHA one-third.

Some PUFAs, including arachidonic acid and EPA together with their metabolites, have important functions as second messengers and neuromodulators (Fenton et al, 2000).

\section{Abnormal phospholipid metabolism in schizophrenia}

The evidence relating to abnormal phospholipid metabolism and schizhophrenia can be summarised as follows (see Box 1 for brief outline).

In 31P magnetic resonance spectroscopy studies of people with schizophrenia, including unmedicated subjects, Keshavan et al (2000) found decreased levels of phosphomonoesters and increased levels of phosphodiesters, particularly in the frontal and temporal lobes. As these reflect phospholipid synthesis and breakdown, respectively, an abnormality of phospholipid metabolism is strongly supported by these findings.

Levels of calcium-independent phospholipase A2 (PLA2) have been shown to be elevated in both the blood and brain of people with schizophrenia. This enzyme is involved in the breakdown of phospholipids by cleaving fatty acids from the Sn2 position (Ross et al, 1999).

Reduced levels of several n3 and n6 PUFAs have been found in cell membranes from erythrocytes and 
Box 1 Evidence that fatty acid metabolism is abnormal in schizophrenia

Reduced fatty acid levels in cell membranes Reduced skin flush response to topical niacin Abnormal electroretinogram

Increased levels of calcium-independent phospholipase A2 in blood and brain

Abnormal 31P magnetic resonance spectroscopy of brain phospholipid

brain of patients with schizophrenia (Peet et al, 1995).

The skin flush in response to topical niacin (nicotinic acid) is much reduced in people with schizophrenia (Ward et al, 1998). Healthy subjects flush when niacin is applied to the skin. This is mediated by prostaglandin D2, which is a cyclooxygenase metabolite of arachidonic acid. These findings imply that the metabolic pathway of arachidonic acid is abnormal in schizophrenia. A reduced inflammatory response, which depends in part on the same pathways, is also manifested by the relative rarity of rheumatoid arthritis in patients with schizophrenia (Oken \& Schulzer, 1999). Such findings emphasise the fact that abnormal fatty acid metabolism in schizophrenia has effects on the whole body, not just on the brain.

An epidemiological study found that international variations in the outcome of schizophrenia, based on World Health Organization figures, showed a strong correlation with the relative amounts of saturated and unsaturated fats in the national diet (Christensen \& Christensen, 1988). A study within a group of schizophrenia patients showed that the level of intake of n3 PUFAs in the normal daily diet was strongly correlated with the severity of positive schizophrenic symptoms: more n3 PUFAs were associated with less severe symptoms (Mellor et al, 1995).

A study of the electroretinogram of patients with schizophrenia showed abnormalities consistent with those produced by experimental depletion of n3 PUFAs in primates (Warner et al, 1999).

\section{Treatment studies using PUFAs in schizophrenia}

Previous studies using n6 PUFAs have produced inconsistent results. On the basis of dietary findings my colleagues and I decided to treat schizophrenia with n3 PUFAs (Mellor et al, 1995). In an initial openlabel pilot study (with no placebo control group), concentrated fish oil was given to 20 patients with symptomatic schizophrenia, in addition to their usual antipsychotic medication. This led to significant improvement in symptoms (Mellor et al, 1995). Fish oil contains two major n3 fatty acids, EPA and DHA.

The next pilot study attempted to distinguish between the possible clinical effects of these two fatty acids by comparing an EPA-enriched oil, a DHAenriched oil and a corn oil placebo in a small group of schizophrenia patients. Again, these supplements were given in addition to existing antipsychotic medication, which was left unchanged during the 3-month study period. The improvement of the EPA group was significantly superior to that of the DHA group, even with the small number of subjects, and EPA was superior also to placebo in a secondary analysis based on percentage improvement. All patients treated with EPA improved and half of them improved by more than $25 \%$ on the Positive and Negative Syndrome Scale (PANSS; Kay et al, 1987) total score (Peet et al, 2001).

Three further studies have investigated the effects of EPA in addition to existing antipsychotic drugs. These used ethyl-EPA, which is a purified preparation. In a multi-centre study carried out in the UK, patients were given 1, 2 or $4 \mathrm{~g}$ of ethyl-EPA in addition to their background antipsychotic medication, which was either typical antipsychotic drugs, atypical antipsychotics or clozapine (Peet \& Horrobin, 2002). Patients on a background medication of clozapine showed a clear and highly significant benefit from having ethyl-EPA added to their treatment regime, with an average improvement of $25 \%$ in PANSS scores. Depression ratings also improved significantly. In contrast, there was little or no benefit when ethyl-EPA was added to other antipsychotic agents. With regard to dose response, it was evident that the $2 \mathrm{~g}$ dose was the most effective and that this effect decreased at the higher $4 \mathrm{~g}$ dosage. This unusual dose-response relationship may be explained on the basis of observed changes in erythrocyte fatty acid levels (which reflect those in the brain). There was a doserelated rise in levels of EPA, but only the $2 \mathrm{~g}$ dose was associated with elevated membrane levels of arachidonic acid, possibly due to inhibition of phospholipase A2 by EPA. The $4 \mathrm{~g}$ dose is large enough to displace arachidonic acid from the membrane. Multiple regression analysis indicated that clinical improvement was best predicted by a rise in membrane arachidonic acid. This is of theoretical interest because arachidonic acid is an important second messenger in the brain (Peet et al, 1994). 
Another double-blind trial of EPA (Fenton et al, 2001), carried out in the USA, involved adding $3 \mathrm{~g}$ of ethyl-EPA to existing antipsychotic medication. This study had a negative outcome, which is perhaps explained by the chronicity of the illness and the relatively high dose of EPA used.

Recently, Emsley et al (2002) reported that $3 \mathrm{~g}$ per day of ethyl-EPA added into existing antipsychotic treatment in a double-blind, placebo-controlled design led to significant reductions in both symptom ratings and severity of tardive dyskinesia.

A double-blind placebo-controlled study of EPA as a sole treatment was carried out by a team in Baroda, India (Peet et al, 2001). They gave EPA or placebo to a group of unmedicated schizophrenia patients and used conventional antipsychotic drugs only if this was clinically imperative. By the end of the 3-month study every patient on placebo required treatment with antipsychotic drugs, whereas in the EPA group half of the patients were maintained on EPA alone and had a better clinical outcome. This double-blind study is supported by two single case reports. Puri et al (2000) described a patient who showed marked improvement on ethyl-EPA that has now been sustained for 3 years. In addition, they reported normalisation of membrane fatty acid levels and even an apparent reversal of cerebral ventricular dilatation. Su et al (2001) reported a patient with an acute exacerbation of schizophrenic symptoms during pregnancy who improved dramatically when treated with $\mathrm{n} 3$ fatty acids as a monotherapy.

\section{Association or causation?}

There is strong evidence of an association between schizophrenia and abnormal phospholipid metabolism. However, association does not prove causation: only intervention studies can achieve that. There are now five published placebo-controlled double-blind trials of EPA in schizophrenia (see Emsley, 2002; Fenton et al, 2001; Peet et al, 2001; Peet \& Harrobin, 2002), four of which have significant positive findings. However, a conservative view is that the present evidence regarding the efficacy of EPA in the treatment of schizophrenia is suggestive but not definitive.

From a research perspective, the most promising approach at present appears to be to use a $2 \mathrm{~g}$ dose of ethyl-EPA to treat patients who either have a background medication of clozapine or take EPA as a sole treatment. The pilot trials used varying methodologies and we now need large well-focused studies. Early studies used heavily flavoured concentrated fish oil; although patients appeared unable to distinguish between treatments, the risk of breaking the double-blind is avoided by using highly purified ethyl-EPA in capsules that do not have a fishy taste or after-taste. There is also interest in the possibility of using ethyl-EPA for early intervention in schizophrenia, at a stage when the diagnosis is uncertain and it would not be appropriate to risk the side-effects of standard antipsychotic medication. Possible use of ethyl-EPA during pregnancy also needs to be studied in a research setting.

From a practical clinical perspective, use of $n 3$ PUFAs is open to debate. On the positive side, they will do no harm: indeed, they are beneficial to physical health and can normalise the elevated triglycerides commonly caused by clozapine (Henderson et al, 2000). On the negative side, the published evidence base for efficacy is relatively weak. The Maudsley hospital's prescribing guidelines (Taylor et al, 2001) suggest considering $\mathrm{n} 3$ triglycerides in the following situations: first, as an addition to clozapine when 3-6 months of clozapine alone have provided no clear benefit; and second, as an adjunctive treatment used as an alternative to clozapine where clozapine has proved toxic or is contraindicated.

\section{Fatty acids and depression}

The evidence suggesting an association between fatty acids and depression is outlined in Box 2 and summarised below.

Data on fish consumption in the national diet have been used to demonstrate correlations between fish consumption and heart disease. Using the same data, highly significant correlations have emerged between fish consumption and rates of depression (Hibbeln, 1998). This relationship is of great interest because of the well-recognised association between depression and heart disease. People with an episode of major depression have a trebled risk of

Box 2 Evidence of an association between n3 fatty acids and depression

Correlation between international variations in fish consumption and rates of depression

DHA content of mothers' milk predicts international variations in post-partum depression

Individuals who are infrequent fish eaters are more likely to become depressed

Preliminary treatment studies suggest efficacy of n3 fatty acids in bipolar disorder and depression 
cardiac mortality later in life (Penninx et al, 2001). It has been suggested that this may be because of a common aetiological factor related to a lack of n3 fatty acids.

The DHA content of mothers' milk (which reflects maternal n3 status) and seafood consumption both predict prevalence rates of post-partum depression across countries (Hibbeln, 2002).

A large study of the Finnish general population showed that the likelihood of having depressive symptoms was significantly higher among infrequent fish consumers (Tanskanen et al, 2001).

There is a well-replicated finding that plasma and red blood cell levels of $\mathrm{n} 3$ fatty acids are reduced significantly in patients with depression, independently of medication status (Edwards et al, 1998). Variations in fatty acid levels correlate with severity of depression.

\section{Treatment studies with $\mathrm{n} 3$ fatty acids in depression}

There have been four published or reported studies of n3 fatty acid treatment of mood disorder. Stoll et al (1999) reported beneficial effects from using highdose fish oil in addition to existing medication for treatment-refractory bipolar disorder. They showed a significant reduction in relapse rate over a 4-month period in the treatment group relative to a placebotreated control group. This work has not yet been replicated. Peet \& Horrobin (2001) have reported a study in primary care in which 1,2 , or 4 g per day of ethyl-EPA or placebo were added to the treatment of patients who had failed to respond to initial antidepressant medication. Patients on ethyl-EPA showed marked improvement relative to those on placebo. Again this appeared to be dose-dependent, with a $1 \mathrm{~g}$ dose producing the biggest improvement and less effect from dosages of 2 and $4 \mathrm{~g}$. In a separate study using DHA, no beneficial effects were found in depression (Marangell, 2000).

Nemets et al (2002) added ethyl-EPA or placebo to maintenance antidepressant therapy in a group of patients with a breakthrough major depresive disorder. They found highly significant benefits from the addition of ethyl-EPA compared with placebo by the end of week 3 of treatment.

Thus, it appears that in depression, as in schizophrenia, EPA may be beneficial, but that DHA has no effect. However, these findings await replication.

Epidemiological evidence relating depression to fish consumption is particularly compelling. Initial double-blind trials of $\mathrm{n} 3$ fatty acid supplementation are promising. However, the initial findings await replication and there is insufficient evidence to recommend $\mathrm{n} 3$ fatty acids as a proven treatment for depression.

\section{Lifestyle and mental health}

The treatment of schizophrenia and depression is commonly viewed from a pharmacological and social perspective, but issues of broader lifestyle are less frequently examined.

Patients with schizophrenia have a variety of enhanced risk factors for cardiovascular disease. These included a sedentary lifestyle, poor diet, smoking and obesity. Furthermore, the treatments we use, apart from having direct effects on the cardiovascular system, can lead to significant weight gain associated with abnormal blood lipid profiles, including elevated triglycerides. This has been shown particularly for clozapine and olanzapine. Promotion of a healthy lifestyle, including exercise, smoking cessation and weight control, will help to minimise these risk factors. More specifically, the elevated triglycerides caused by atypical antipsychotic drugs appear be reversible by the use of n3 PUFAs. The mean triglyceride level in patients with schizophrenia is elevated to above the normal range by clozapine and significantly reduced to within the normal range by 2 or $4 \mathrm{~g}$ of ethyl-EPA (Peet \& Horrobin, 2001). Omega-3 PUFAs reduce triglyceride levels by inhibiting their synthesis in the liver (Connor et al, 1993). Although a reduction in triglyceride levels might be achieved to some extent by improving diet there are some patients who would benefit physically from n 3 PUFA supplementation irrespective of any potential benefits to the mental state.

Similar considerations apply to states of depression. Not only are cardiovascular risk factors increased in depression, but there is strong epidemiological evidence linking depression with heart disease. The link between heart disease itself and fish consumption has been recognised for several decades, but this is usually dealt with by advice on diet rather than recommending supplements. If the current data on depression are to be believed, then dietary modification, including increased fish consumption, should reduce the risk for depression as well as for heart disease. Similarly, physical exercise not only reduces the risk of heart disease but also alleviates depression (Scully et al, 1998).

One very positive aspect of work on n3 PUFAs and mental illness is that the proposed interventions, particularly when taken into a broader lifestyle 
context, are those that we should in any case be recommending to our patients as part of their general health and welfare.

\section{References}

Christensen, O. \& Christensen, E. (1988) Fat consumption and schizophrenia. Acta Psychiatrica Scandinavica, 78, 587591.

Connor, W. E., De Francesco, C. A. \& Connor, S. L. (1993) N3 fatty acids from fish oil. Effects on plasma lipoproteins and hypertriglyceridemic patients. Annals of the New York Academy of Science, 683, 16-34.

Edwards, R., Peet, M., Shay, J., et al (1998) Omega-3 polyunsaturated fatty acid levels in the diet and in red blood cell membranes of depressed patients. Journal of Affective Disorders, 48, 149-155.

Emsley, R. A., Myburgh, C. C., Oosthuizen, P. P., et al (2002) Omega-3 fatty acid and schizophrenia: a randomised trial of ethyl-eicosapentaenoic acid (ethylEPA) versus placebo as add-on treatment. Schizophrenia Research, 53, 9.

*Fenton, W. S., Hibbeln, J. \& Knable, M. (2000) Essential fatty acids, lipid membrane abnormalities, and the diagnosis and treatment of schizophrenia. Biological Psychiatry, 47, 8-21.

- Dickerson, F., Boronow, J., et al (2001) A placebo-controlled trial of omega-3 fatty acid (ethyl eicosapentaenoic acid) supplementation for residual symptoms and cognitive impairment in schizophrenia. American Journal of Psychiatry, 158, 2071-2074.

Henderson, D. C., Cagliero, E., Gray, C., et al (2000) Clozapine, diabetes mellitus, weight gain and lipid abnormalities: a five year naturalistic study. American Journal of Psychiatry, 157, 975-981.

*Hibbeln, J. R. (1998) Fish consumption and major depression. Lancet, 352, 71-72.

- (2002) Seafood consumption, the DHA content of mother's milk and prevalence rates of postpartum depression: a cross-national, ecological analysis. Journal of Affective Disorders, in press.

Kay, S. R., Fiszbein, A \& Opler, L. A. (1987) The positive and negavite syndrome scale (PANSS) for schizophrenia. Schizophrenia Bulletin, 13, 261-276.

Keshavan, M. S., Stanley, J. A. \& Pettegrew, J. W. (2000) Magnetic resonance spectroscopy in schizophrenia: methodological issues and findings - Part II. Biological Psychiatry, 48, 369-380.

Marangell, L. B., Zboyan, H. A., Cress, K. K., et al (2000) A double-blind placebo-controlled study of decosahaexanoic acid in the treatment of depression. Inform, 11, S78.

Mellor, J. E., Laugharne, J. D. \& Peet, M. (1995) Schizophrenic symptoms and dietary intake of n3 fatty acids. Schizophrenia Research, 18, 85-86.

Nemets, B., Stahl, Z. \& Belmaker, R. H. (2002) Addition of omega-3 fatty acid to maintenance medication treatment for recurrent unipolar depressive disorder. American Journal of Psychiatry, 159, 477-479.

Oken, R. J. \& Schulzer, M. (1999) At issue: schizophrenia and rheumatoid arthritis: the negative association revisited. Schizophrenia Bulletin, 25, 625-638.

Peet, M. \& Horrobin, D. F. (2001) A dose-ranging study of ethyl-eicosapentonoate in treatment-unresponsive depression. Journal of Psychopharmacology, 15(Suppl.), A12.

— \& - (2002) A dose-ranging exploratory study of the effects of ethyl-eicosaepentonate in patients with persistent schizophrenic symptoms. Journal of Pyschiatric Research, 36, 7-18.

- Laugharne, J. D., Horrobin, D. F., et al (1994) Arachidonic acid: a common link in the biology of schizophrenia? Archives of General Psychiatry, 51, 665-666.
- - - Rangarajan, N., et al (1995) Depleted red cell membrane essential fatty acids in drug treated schizophrenic patients. Journal of Psychiatric Research, 29, 227-232.

* _ Brind, J., Ramchand, C. N., et al (2001) Two double blind placebo-controlled pilot studies of eicosapentaenoic acid in the treatment of schizophrenia. Schizophrenia Research, 49, 243-251.

Penninx, B. W., Beekman, A. T., Horig, A., et al (2001) Depression and cardiac mortality: results from a community based longitudinal study. Archives of General Psychiatry, 58, 229-230.

Puri, B. K., Richardson, A. J., Horrobin, D. F., et al (2000) Eicosapentaenoic acid treatment in schizophrenia associated with symptom remission, normalisation of blood fatty acids, reduced neural membrane phospholipid turnover and structural brain changes. International Journal of Clinical Practice, 54, 57-63.

Ross, B. M., Turenne, S., Mosczynska, A., et al (1999) Differential alteration of phospholipase A2 activities in brains of patients with schizophrenia. Brain Research, 821, 407-413.

Scully, D., Kremer, J., Meade, M. M., et al (1998) Physical exercise and psychological well-being: a critical review. British Journal of Sports Medicine, 32, 111-120.

*Stoll, A. L., Severus, W. E., Freeman, M. P., et al (1999) Omega-3 fatty acids in bipolar disorder: a preliminary double blind, placebo controlled trial. Archives of General Psychiatry, 56, 407-412.

Su, K. P., Shen, W. W. \& Huang, S. Y. (2001) Omega-3 fatty acids as a psychotherapeutic agent for a pregnant schizophrenic patient. European Neuropsychopharmacology, 11, 295-299.

Tanskanen, A., Hibbeln, J. R., Tuomilehto, J., et al (2001) Fish consumption and depressive symptoms in the general population in Finland. Psychiatric Services, 52, 529-531.

Taylor, D., McConnell, H., Duncan-McConnell, D., et al (2001) The South London and Maudsley NHS Trust 2001 Prescribing Guidelines. London: Martin Dunitz.

*Ward, P. E., Sutherland, J., Glenn, E. M., et al (1998) Niacin skin flush in schizophrenia: a preliminary report. Schizophrenia Research, 29, 269-274.

Warner, R., Laugharne, J., Peet, M., et al (1999) Retinal function as a marker for cell membrane omega-3 fatty acid depletion in schizophrenia: a pilot study. Biological Psychiatry, 45, 1138-1142.

* indicates articles of particular interest

\section{Multiple choice questions}

1. Polyunsaturated fatty acids:

a are essential constituents of cell membrane phospholipid

b can all be synthesised by the body

c make up $15-30 \%$ of the dry weight of the brain

$\mathrm{d}$ have no functional importance in the brain

e are important in cell signalling.

2. The following fatty acids are metabolised to eicosanoids:

a eicosapentaenoic acid

b linoleic acid

c arachidonic acid

d docosahexaenoic acid

e dihomogamma linolenic acid. 
3. Abnormal fatty acid metabolism in schizophrenia is indicated by:

a reduced fatty acid levels in red blood cell membranes

b dysfunction of smooth pursuit eye movement

c abnormal 31P magnetic resonance spectroscopy

$\mathrm{d}$ reduced skin flush response to niacin

e elevated triglycerides during clozapine treatment.

4. An association between $\mathrm{n} 3$ fatty acids and depression is suggested by:

a correlation between international variations in fish consumption and depression

b treatment studies indicating possible efficacy of $\mathrm{n} 3$ fatty acids in bipolar disorder and depression

c low rates of heart disease in depression

$\mathrm{d}$ reduced levels of $\mathrm{n} 3$ fatty acids in cell membranes of patients with unmedicated depression

e effectiveness of exercise in alleviating depression.
5. Eicosapentaenoic acid (EPA):

a is mostly derived from fish in the diet

b is an inhibitor of phospholipase A2

c causes elevation of blood triglycerides

d is a major constituent of brain phospholipid

e has shown preliminary evidence of efficacy in schizophrenia and depression.

\begin{tabular}{|c|c|c|c|c|}
\hline \multicolumn{5}{|c|}{ MCQ answers } \\
\hline 1 & 2 & 3 & 4 & 5 \\
\hline a $T$ & a $T$ & a $T$ & a $T$ & a $T$ \\
\hline b $\mathbf{F}$ & b $F$ & b $\mathbf{F}$ & b $T$ & b $T$ \\
\hline c $T$ & c $\mathbf{T}$ & c $\mathrm{T}$ & c $F$ & c $F$ \\
\hline d F & d F & d $T$ & d $T$ & d F \\
\hline e $\mathrm{T}$ & e $\mathbf{T}$ & e $\mathbf{F}$ & e $\mathrm{F}$ & e $\mathbf{T}$ \\
\hline
\end{tabular}

